\title{
Participation accrue des personnes vivant avec le VIH/SIDA aux activités des ONG de Lutte Contre le SIDA
}

Population Council

Follow this and additional works at: https://knowledgecommons.popcouncil.org/departments_sbsr-hiv How does access to this work benefit you? Let us know!

\section{Recommended Citation}

"Participation accrue des personnes vivant avec le VIH/SIDA aux activités des ONG de Lutte Contre le SIDA," Horizons Research Summary. Washington, DC: Population Council, 2003. 


\section{Hqrizons $\Lambda$}

\section{PARTICIPATION ACCRUE DES PERSONNES VIVANT AVEC LE VIH/SIDA AUX ACTIVITÉS DES ONG DE LUTTE CONTRE LE SIDA}

\section{Résultats d'une étude réalisée dans quatre pays}

$\mathrm{L}$

ors de la conclusion du Sommet sur le SIDA qui s'est tenu à Paris en 1994, 42 gouvernements ont signé une déclaration en faveur d'une participation accrue des personnes vivant avec le VIH/SIDA (PVVIH) à la formulation des politiques de lutte contre le SIDA et à la prestation des services offerts par les organisations non gouvernementales (ONG). Alors qu'on reconnaît de plus en plus son importance, il n'existe pourtant que peu de travaux de recherche qui se sont penchés sur la participation des PVVIH dans les activités de prévention et de prise en charge des associations de lutte contre le SIDA dans des pays en développement et son impact sur les PVVIH, d'autres personnes affectées par le VIH/SIDA et les ONG.

Visant à combler cette lacune, le Programme Horizons et l'Alliance internationale contre le VIH/SIDA ont mené une étude sur la participation des PVVIH aux activités des ONG, au Burkina Faso, en Equateur, en Zambie et dans l'état de Maharashtra en Inde. L'étude qui s'est déroulée d'octobre 1998 à

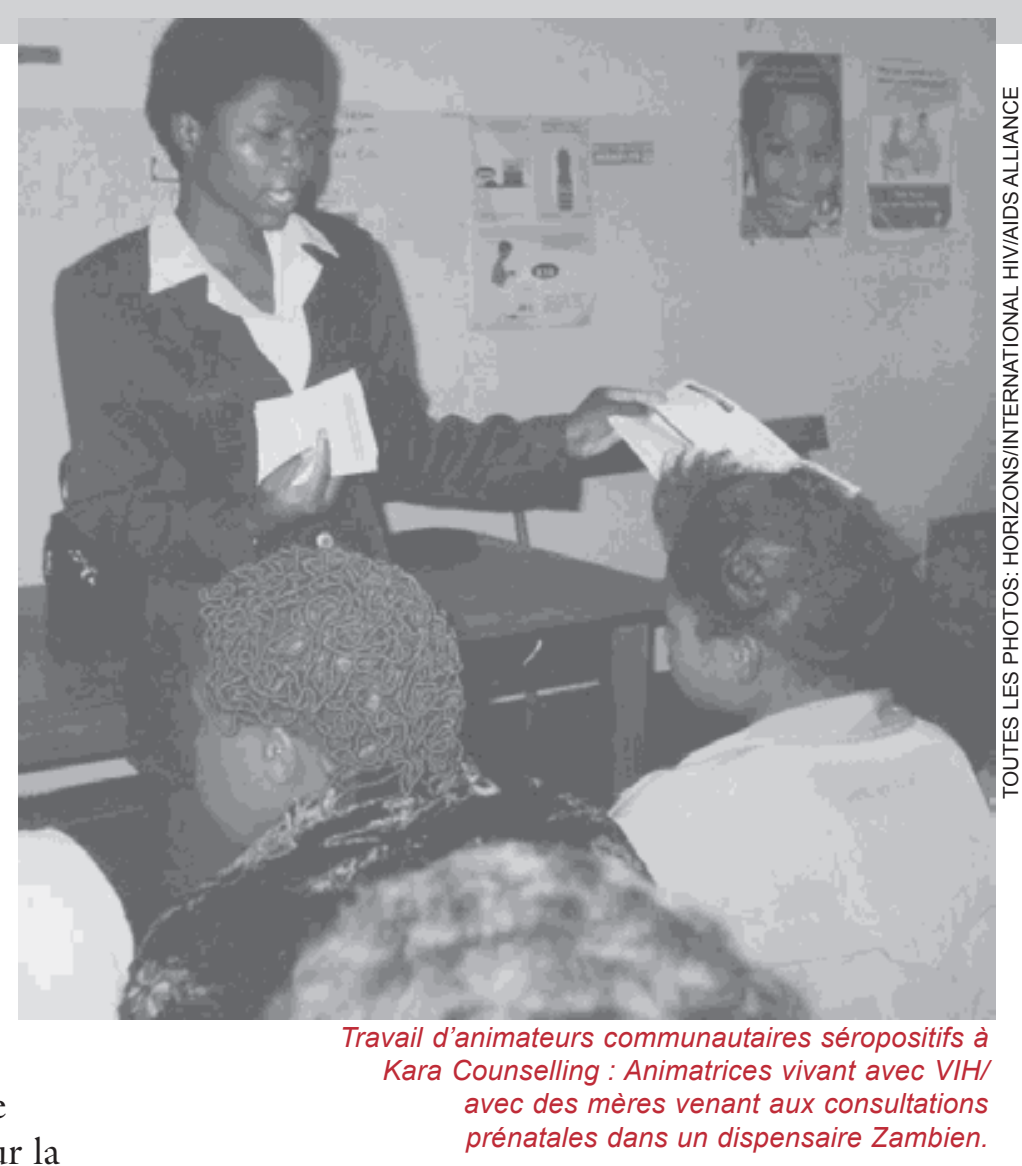

août 2001 avait pour objet de déterminer les conditions qui favorisent la participation des PVVIH et les stratégies que peuvent utiliser les organisations pour parvenir à une véritable participation des PVVIH.

Dix-sept ONG ont participé à l'étude. Toutes mènent des activités de prévention et de prise en charge. Elles représentent différents types d'organisations et différents niveaux de participation des PVVIH. Toutes avaient exprimé leur intérêt pour les objectifs de l'étude. 
ONG ayant participé à

l'étude

- Burkina Faso : Association African Solidarité (AAS) ;

Association Laafi la Viim (ALAVI) ; Appui Moral Matériel et Intellectuel à l'Enfant (AMMIE) ; la Bergerie-Foi, Univers, Compassion ; Association Responsabilité Espoir Vie Solidarité + (REVS+).

- Equateur : Fundación Dios;

Vida y Esperanza ; Fundación Esperanza ; Fundación Siempre Vida ; Fundación Vivir.

- Etat de Maharashtra, Inde : Projet CHILD de Committed Communities Developement Trust (CCDT) ; Maharashtra Network for Positive People (MNP+) ; Programme de développement communautaire VIH/SIDA de l'Armée du Salut Mumbai ; Society of Friends of Sassoon Hospitals (SOFOSH).

- Zambie : Copperbelt Health Education Project (CHEP) ; Hope Humana People to People ; Kara Counselling and Training Trust ; Hôpital de la Mission Chikankata de l'Armée du Salut.

\section{Méthodologie de l'étude}

Dans chacun des quatre pays, des chercheurs ont collecté des données en utilisant des méthodes qualitatives et quantitatives, notamment des entretiens individuels et collectifs, des groupes de discussion dirigée, des observations, un questionnaire pour recueillir des données statistiques sur les services et les prestataires de services. 745 participants ont été recrutés pour l'étude : des utilisateurs de services, des employés et des volontaires des ONG aussi bien séronégatifs que séropositifs, ainsi que des informateurs clés tels que des décideurs de politiques de santé, des professionnels de santé et des leaders communautaires. Dans les quatre pays, les PVVIH participaient aux activités des ONG impliquées dans l'étude, soit en tant que prestataires de services, soit comme utilisateurs des services ou bien les deux.

Les PVVIH représentaient environ la moitié des participants de l'étude en Inde et en Zambie, 35\% en Equateur et $15 \%$ au Burkina Faso. Le tableau 1 récapitule le nombre d'ONG qui ont participé à l'étude ainsi que les sites de l'étude et les types et nombres de participants par pays.

Tout au long de l'étude, les équipes de recherche ont employé une approche à caractère participatif. En effet, dans chaque pays les ONG ont pris part activement à la conception de l'étude, sa mise en ouvre ainsi qu'à la validation et à l'interprétation des résultats. Grâce à un

\section{Diverses formes de participation des PVVIH}

Les équipes de recherche ont utilisé les critères suivants pour examiner le type de participation des PVVIH aux activités des ONG.

- Temps consacré aux activités et régularité de la participation aux activités

- Rémunération : financière (salaires, indemnités, remboursement des frais de déplacement), matérielle (médicaments, nourriture), technique (formation) ou psychologique (soutien social et conseils)

- Compétences utilisées

- Renforcement de compétences assuré par l'organisation

- Portée et autonomie de la prise de décisions

- Niveau de visibilité : interne (au sein de l'ONG) et externe (à l'extérieur de l'ONG) 
Tableau 1 Caractéristiques des sites de l'étude et des populations

\begin{tabular}{|c|c|c|c|c|c|c|}
\hline \multirow[b]{2}{*}{ Pays } & \multirow[b]{2}{*}{$\begin{array}{c}\text { Nombre } \\
\text { d'ONG }\end{array}$} & \multirow[b]{2}{*}{$\begin{array}{l}\text { Sites de } \\
\text { l'étude }\end{array}$} & \multicolumn{4}{|c|}{ Participants à l'étude } \\
\hline & & & $\begin{array}{l}\text { Prestataires } \\
\text { de services } \\
\text { (personnel } \\
\text { des ONG ou } \\
\text { volontaires) }\end{array}$ & $\begin{array}{l}\text { Utilisateurs } \\
\text { de services }\end{array}$ & $\begin{array}{l}\text { Famille des } \\
\text { prestataires } \\
\text { séropositifs }\end{array}$ & $\begin{array}{l}\text { Informateurs } \\
\text { clés }\end{array}$ \\
\hline Burkina Faso & 5 & $\begin{array}{l}1 \text { urbain et } \\
\text { semi-rural } \\
4 \text { urbains }\end{array}$ & 103 & 19 & 5 & 49 \\
\hline Equateur & 4 & 4 urbains & *115 & & 5 & 52 \\
\hline Inde & 4 & $\begin{array}{l}1 \text { urbain et } \\
\text { semi-rural } \\
3 \text { urbains }\end{array}$ & 48 & 82 & 2 & 8 \\
\hline Zambie & 4 & $\begin{array}{l}1 \text { rural } \\
1 \text { urbain et } \\
\text { semi-rural } \\
2 \text { urbains }\end{array}$ & 101 & 115 & 7 & 34 \\
\hline
\end{tabular}

*En Equateur, les chercheurs n'ont pas fait la différence entre prestataires de services et utilisateurs de services car un grand nombre de prestataires séropositifs sont également des utilisateurs de services.

atelier d'information et de consultation tenu au départ de l'étude, les ONG ont pris connaissance des concepts fondamentaux de la recherche, discuté du bien-fondé, de la pertinence et du déroulement prévu de l'étude et adapté la méthodologie et les outils au contexte national. Par ailleurs, dans chaque pays les équipes ont organisé un atelier d'analyse et d'interprétation des données avec les ONG participantes afin de discuter des résultats et, partant de là, de déterminer les stratégies permettant d'accroître la participation des PVVIH.

\section{Contexte des pays dans lesquels l'étude a été réalisée}

D'après les estimations, la prévalence du VIH au sein de la population varie grandement entre les quatre pays (Equateur, moins de 1\% ; Burkina Faso, 6,5\% ; Zambie 20\%). Dans l'état de Maharashtra en Inde, presque $2 \%$ des femmes qui se rendent aux consultations prénatales sont infectées, chiffre révélateur de l'incidence au sein de la population prise dans son ensemble. Dans les deux pays africains, la transmission est essentiellement hétérosexuelle alors qu'en Equateur, la transmission par voie homosexuelle et bisexuelle est responsable d'un tiers des cas de SIDA. En Inde, l'infection se transmet surtout par voie hétérosexuelle bien qu'il existe deux sous-épidémies importantes parmi les hommes qui ont des relations sexuelles avec les hommes ( $\mathrm{HSH}$ ) et les utilisateurs de drogues par voie intraveineuse. Plus de la moitié des PVVIH au Burkina Faso et en Zambie sont des femmes alors que cette proportion s'élève à $35 \%$ en Equateur et en Inde.

Les PVVIH sont victimes de discrimination et de stigmatisation dans les quatre pays à cause de l'association entre le VIH/SIDA et certains comportements et groupes de population. La crainte d'être discriminé ou stigmatisé fait que peu de personnes révèlent leur statut VIH ou même se font dépister pour connaître leur statut. 


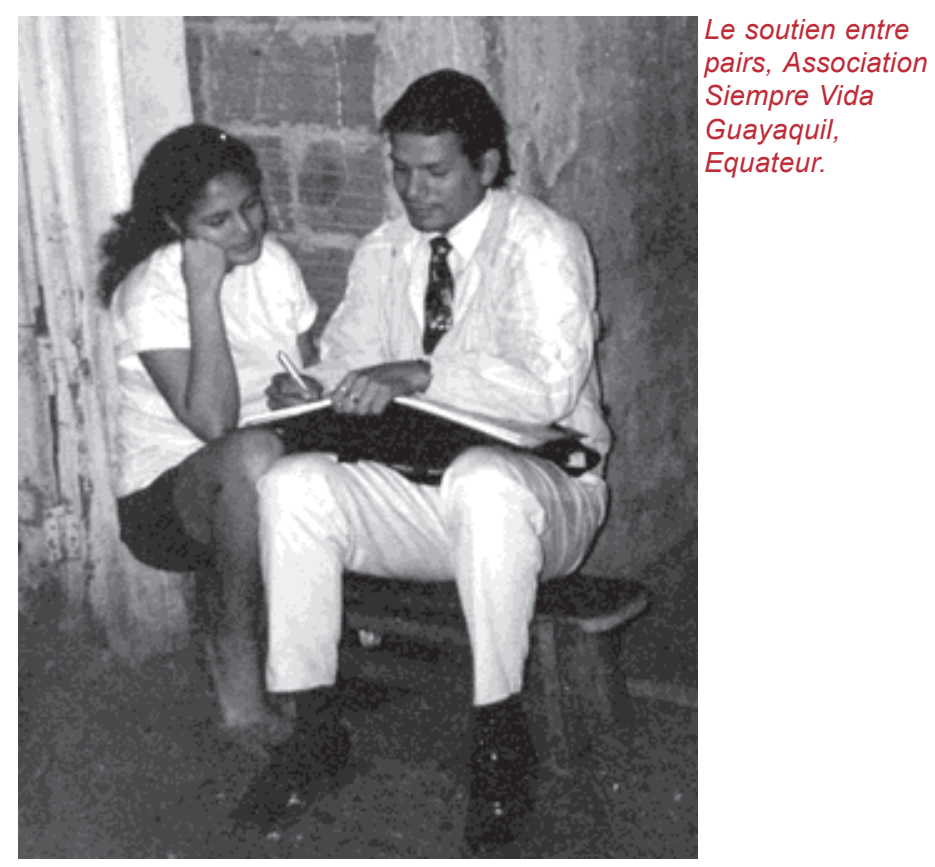

Au Burkina Faso, en Inde et en Zambie, les services de counseling et dépistage volontaire ne sont pas suffisamment disponibles et seules les PVVIH qui ont de l'argent ou des contacts internationaux ont accès aux antirétroviraux. En Equateur, les ARV sont disponibles pour ceux ayant accès aux hôpitaux militaires, et dans une seule région pour les personnes couvertes par la sécurité sociale. L'accès au traitement des infections opportunistes est particulièrement difficile et problématique au Burkina Faso, en Inde et dans les zones rurales en Zambie.

Il existe certes des réseaux nationaux de PVVIH en Equateur, en Inde et en Zambie mais la participation des PVVIH n'est guère visible dans ces quatre pays. C'est à la fin des années 80 et au début des années 90 que les PVVIH ont commencé à se mobiliser en Equateur et en Zambie. En revanche le phénomène est relativement récent au Burkina Faso et en Inde. C'est essentiellement à l'initiative des bailleurs de fonds, des ONG et des activistes internationaux que la participation des PVVIH s'est accrue dans les quatre pays concernés. En effet, ce sont eux qui ont soutenu le développement des organisations et des réseaux de PVVIH et ont encouragé les gouvernements à accorder une plus grande priorité à la participation des PVVIH pour lutter contre l'épidémie.

\section{Principaux résultats}

\section{Il existe quatre types distincts de participation des PVVIH.}

L'accès aux services pour les PVVIH suppose la participation à des activités des ONG en tant que bénéficiaires ou utilisateurs de services tels que les soins médicaux, le counseling ou la formation. L'accès est le type le plus courant de participation des PVVIH qui a été observé parmi les 17 ONG participant à l'étude. A ce premier niveau, les PVVIH peuvent non seulement acquérir des connaissances sur le VIH/SIDA mais aussi apprendre à accepter leur statut VIH et à affronter l'infection, ce qui peut les motiver pour aider d'autres personnes.

Je me sentais tellement seul. Je pensais que j'allais mourir sur place...le groupe...m'a apporté beaucoup de soutien psychologique... je ne suis pas allé voir un psychologue mais ce qui m'aidé, c'est d'être avec un groupe de personnes qui se trouvaient dans la même situation. Cela m'a redonné confiance et j'ai retrouvé l'estime de moi-même.

Membre séropositif, Fundación Siempre Vida, Equateur

Voici des exemples de services qui visent à donner aux PVVIH les moyens d'agir et partant à encourager une participation accrue aux activités des ONG :

- Les formations sur comment mener une vie positive avec le VIH aident les PVVIH à accepter leur statut et leur apportent des informations et des compétences pour prendre en main leur existence.

- Les groupes de parole sont l'occasion de partager des problèmes, de s'apporter une aide mutuelle et sont un espace protégé où les PVVIH peuvent révéler leur statut. Les groupes de parole renforcent l'estime que les PVVIH ont d'elles-mêmes et peuvent les préparer à parler de leur situation à l'extérieur du groupe.

- Les séances de counseling aident les PVVIH à accepter leur statut et les préparent aux répercussions possibles de la visibilité. Les conseillers peuvent orienter les PVVIH vers 


\section{Mobilisation et participation des PVVIH}

Selon les données recueillies, les PVVIH sont mobilisées de différentes manières :

Dans cinq ONG, les PVVIH ont mis sur pied les organisations, souvent en collaboration avec d'autres personnes affectées, des professionnels de santé ou des travailleurs sociaux. Ces ONG sont des organisations de défense des droits des PVVIH et/ou des groupes d'autosupport. Les modèles de participation sont essentiellement la participation accrue et l'implication, bien qu'il y ait également des PVVIH qui utilisent les services. Ces organisations changent en fonction de la composition des membres. Dans le cas de deux ONG, les membres fondateurs séropositifs sont décédés et les organisations se sont peu à peu consacré plus à la prestation de services et moins à la défense des droits des PVVIH.

Dans des ONG sans participation visible de PVVIH au départ, par exemple dans des organisations caritatives, religieuses ou laïques, et des ONG de développement, le processus fonctionne de différentes manières :

- Les ONG donnent les moyens d'agir et les compétences nécessaires à leurs usagers qui sont séropositifs pour qu'ils deviennent prestataires de services, selon le modèle de l'inclusion et, parfois, de l'implication, comme ceci a été observé dans six ONG. Cela débouche rarement sur la participation accrue des PVVIH à moins qu'il n'existe une politique délibérée de l'organisation comme on a pu le voir dans une ONG en Zambie.

- Les associations recrutent des PVVIH qui ne sont pas des utilisateurs de leurs services et qui sont visibles. Ces PVVIH disposent déjà de l'expérience et des compétences leur permettant d'être prestataires de services ou de faire partie du management de l'organisation (implication et participation accrue). Cette situation a par exemple été observée dans une ONG de développement en Zambie.

- Parfois, un employé ou un volontaire d'ONG découvre qu'il est séropositif alors qu'il travaille déjà dans une organisation et il décide de révéler son statut sérologique. Toutefois, une telle situation ne s'est présentée que dans une seule organisation au Burkina Faso. d'autres activités des ONG et vers des formations pour renforcer leurs capacités afin qu'ils puissent participer plus pleinement.

Lors du counseling...ils découvrent leur valeur personnelle au sein de la société et dans la communauté dont ils proviennent. Les choses qu'ils apprennent lors du counseling, l'éducation qu'ils reçoivent et le partage de leurs propres expériences les aident à révéler leur situation pour pouvoir aider la communauté.

Prestataire de service, Kara Counselling and Training Trust, Zambie

L'inclusion, dans ce cas, l'ONG recrute les PVVIH en tant que personnel peu qualifié ou bénévoles dans le cadre d'activités qui ne sont pas directement liées au VIH/SIDA, pour faire le ménage ou la cuisine par exemple. Les PVVIH peuvent aussi participer de manière bénévole, informelle et occasionnelle à des activités comme les groupes de parole, les visites à domicile ou à l'hôpital ou des actions de sensibilisation. A ce niveau, la formation formelle des PVVIH reste limitée. Les contacts avec d'autres PVVIH sont parmi les motivations les plus importantes pour les PVVIH. En revanche, il n'existe que peu de récompenses matérielles pour leurs efforts. Du point de vue de l'ONG, il peut être rentable d'utiliser des PVVIH comme bénévoles, mais cela peut avoir des effets négatifs si ces bénévoles ne sont pas bien formés.

L'implication. Dans ce cas, les PVVIH dispensent des services liés au VIH/ SIDA de manière formelle et régulière en tant qu'employés ou volontaires. Leurs compétences sont reconnues par l'organisation et ils touchent généralement une rémunération financière en contrepartie de leur travail, 
bien qu'au Burkina Faso et en Equateur, un grand nombre de volontaires réguliers ne soient pas payés. Ces PVVIH participent à la planification des services qu'ils fournissent et, dans certaines organisations, on les consulte à propos d'autres services.

A ce niveau de participation, les PVVIH ont accepté leur statut et utilisent leur expérience de vie avec le VIH/SIDA ainsi que les compétences et connaissances théoriques acquises lors de la formation pour dispenser des services.

La participation accrue A ce stade, les PVVIH prennent part à la gestion d'une organisation, à la planification de ses activités et à la définition de ses stratégies en tant que directeurs, membres du conseil d'administration ou responsables de programmes. Elles peuvent représenter l'organisation à l'extérieur. Ce type de participation suppose une plus grande visibilité des PVVIH qui risque de les exposer à la stigmatisation et à la discrimination, bien que ces PVVIH soient mieux équipées et mieux préparées à y faire face en raison d'un niveau plus élevé d'acceptation personnelle et de soutien.

\section{La participation accrue des PVVIH a été} observée dans des organisations qui ont pour vocation de défendre les droits des personnes

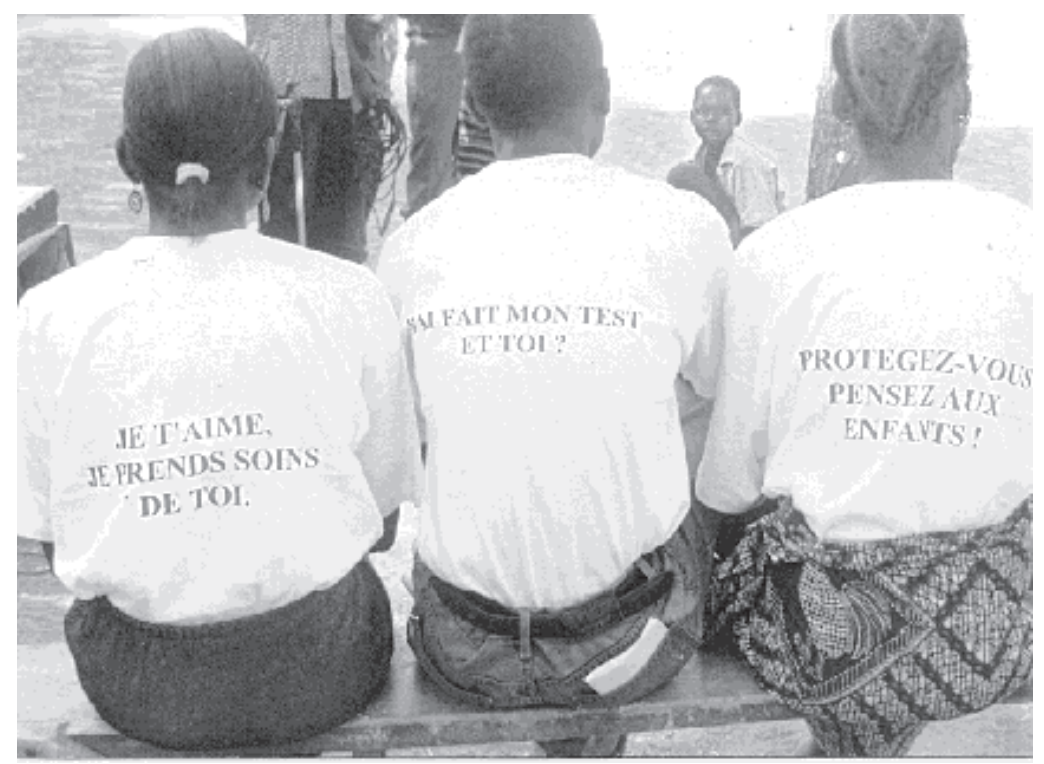

Les membres de REVS+ au Burkina Faso portent des T-shirts avec des messages de prévention lors d'une campagne de sensibilisation sur le VIH/SIDA. séropositives, telles que les groupes et réseaux de PVVIH. A une exception près, on remarque que les PVVIH prenant part à la gestion d'une $\mathrm{ONG}$, à la planification de ses activités et à la définition de ses stratégies ont au minimum une instruction secondaire et appartiennent aux classes moyennes. La plupart participent depuis des années à la lutte contre l'épidémie et ont reçu une formation considérable. Voici des exemples de ce type de participation :

- A MNP+ en Inde et à REVS+ au Burkina Faso, ce sont des PVVIH qui gèrent l'organisation et certains programmes et, à CHEP en Zambie, le coordinateur et l'assistant du programme pour la participation des PVVIH sont tous les deux séropositifs.

- A MNP+ et à REVS+, les PVVIH sont des membres (volontaires) du conseil d'administration. A CHEP et à Kara Counselling and Training Trust en Zambie, les coordinateurs de programme séropositifs participent à la définition des stratégies de l'organisation dans laquelle ils travaillent.

Les PVVIH qui gèrent les organisations et les programmes occupent souvent une position importante à l'extérieur de l'ONG où elles travaillent, exprimant les points de vue et devenant les porte-parole des PVVIH dans différents forums. Un grand nombre d'entre elles participent à des activités de plaidoyer dans les réseaux nationaux et régionaux de PVVIH.

\section{La participation aux activités des ONG peut avoir un rôle thérapeutique pour les PVVIH.}

Bien que l'impact de la participation des PVVIH dépende de la manière dont elles sont impliquées dans une $\mathrm{ONG}$, les personnes interrogées durant l'étude ont indiqué dans l'ensemble que la participation a un rôle thérapeutique. L'état psychologique s'améliore grâce à un soutien accru des pairs et une meilleure connaissance du VIH. Les individus sont en général moins isolés. L'état physique est également souvent meilleur car les PVVIH 
ont plus d'informations sur les traitements, elles savent notamment où s'adresser et comment s'y prendre pour avoir accès aux traitements. Dans certains cas la situation matérielle des PVVIH progresse en raison de la rémunération ou des avantages matériels qu'elles reçoivent en échange de leur travail.Certaines personnes en Zambie et en Equateur ont également expliqué qu'elles avaient adopté des comportements moins risqués, en particulier la réduction du nombre de partenaires sexuels. D'autres ont cité une meilleure intégration des PVVIH au sein de leur famille lorsque celles-ci réalisent qu'elles peuvent avoir une activité productive. Par ailleurs les PVVIH peuvent informer leurs parents sur le VIH/SIDA.

La famille souhaite que j'aide mes jeunes parents...et donc ma famille est très heureuse que je ne dissimule pas mon état.

Prestataire de service séropositif, CHEP, Zambie

Ils me connaissent à l'hôpital et les portes me sont toujours ouvertes. Je vais là-bas et je m'assure qu'ils s'occupent de mes patients.

Conseiller séropositif, Siempre Vida, Equateur

Le groupe m'a vraiment remonté le moral... lorsque nous nous rencontrons dans le groupe, nous nous sentons mieux car nous sommes unis telle une famille. On est un soutien pour ceux qui viennent juste d'être dépistés.

Participant d'un groupe d'entraide de PVVIH, CHEP, Zambie

L'avantage c'est qu'en rejoignant

l'association, j'ai appris tant de choses. Si j'étais resté enfermé à la maison, je ne saurais pas tant de choses.

Prestataire de services séropositif, $\mathrm{MNP}+$, Inde

Toutefois, les personnes interviewées dans les quatre pays ont également mentionné des inconvénients ou difficultés soulevés par la participation des PVVIH, en fonction des activités réalisées et du niveau de visibilité. Par exemple, les PVVIH asymptomatiques impliquées dans la prise en charge peuvent recevoir des chocs psychologiques lorsqu'elles sont en contact étroit avec des personnes très malades. En Equateur et en Zambie, certaines PVVIH ont été victimes de discrimination et de stigmatisation à cause de leur participation visible aux activités d'associations de lutte contre le SIDA. Quelques personnes interrogées au Burkina Faso et en Zambie ont dit qu'il était même arrivé que des gens accusent les PVVIH de prétendre qu'elles sont séropositives pour obtenir de l'argent des bailleurs de fonds. Les ONG doivent être conscientes des effets négatifs possibles de la participation pour les limiter.

J'ai beaucoup souffert de discrimination depuis que j'ai fait savoir la vérité et surtout depuis que j'ai décidé de participer aux activités. Les gens se moquent de moi et mes amis ne veulent plus jouer au football avec moi...mes beaux-parents ont tout fait pour que ma femme me quitte et ils ont réussi.

Prestataire de service séropositif, Armée du Salut, Zambie

\section{La participation des PVVIH renforce les ONG.}

Les données de l'étude montrent que la participation des PVVIH à la prestation de services et à la gestion des ONG aide à améliorer les services et à renforcer les organisations de la façon suivante :

- Amélioration des services de prise en charge qui deviennent mieux adaptés aux besoins des PVVIH et plus personnalisés.

- Efficacité accrue des activités de prévention en améliorant la manière dont les gens perçoivent les PVVIH.

- Sensibilisation des employés des ONG aux problèmes des PVVIH par la prise en compte de leur point de vue dans les organisations et en encourageant le personnel à reconnaître la contribution faite par les PVVIH.

- Plus grande crédibilité de l'organisation et de ses services.

- Champ d'action élargi avec inclusion d'activités de plaidoyer pour défendre les droits des PVVIH. 


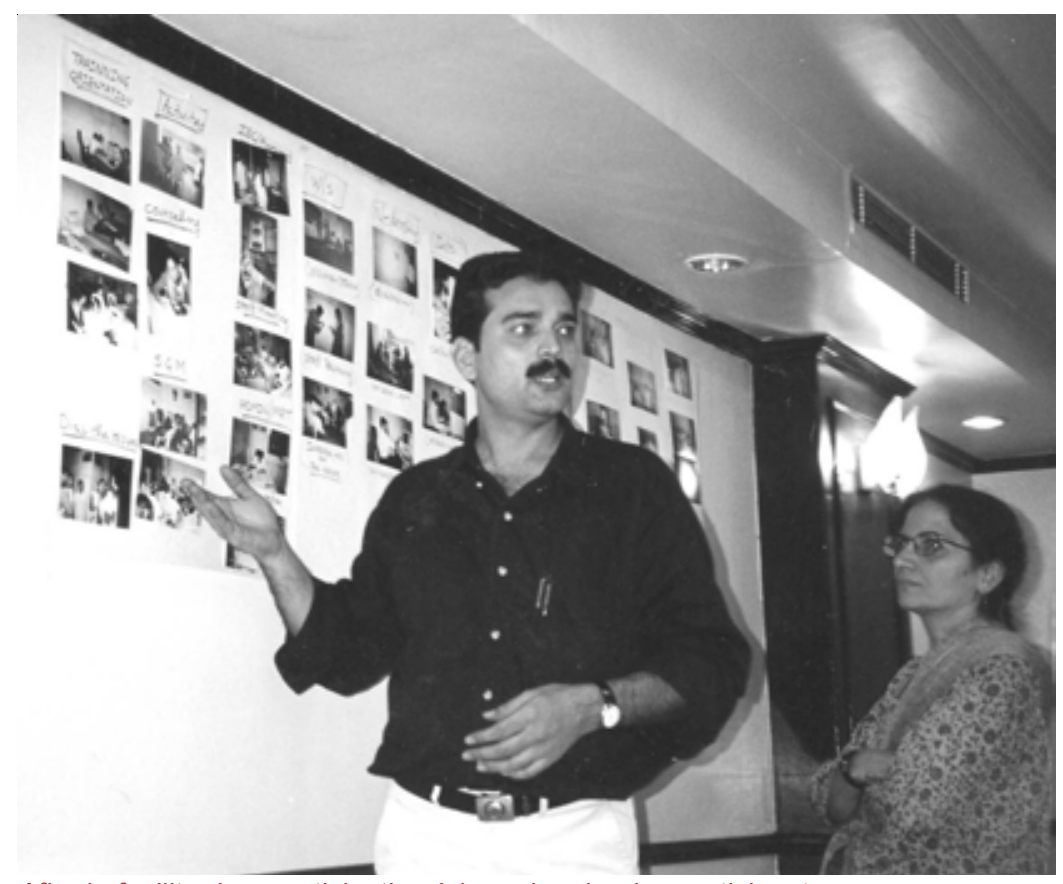

Afin de faciliter leur participation à la recherche, les participants de l'étude utilisent des photographies pour discuter de la participation des PVVIH aux activités des associations lors d'un atelier d'analyse de données en Inde.

Je me suis rendu compte qu'il s'agissait de gens normaux comme vous et moi. Je peux manger avec eux et partager les mêmes plats. A présent, je comprends bien mieux le VIH qu'avant.

Prestataire de service séronégatif, Kara Counselling and Training Trust, Zambie

En outre, les personnes interviewées ont fait mention de plusieurs effets négatifs que peut avoir la participation des PVVIH pour une organisation. Toutefois, les répercussions négatives de la participation des PVVIH peuvent être atténuées si elles sont prises en compte en amont par les associations. Par exemple, des PVVIH qui ne sont pas suffisamment formées risquent de communiquer des informations inexactes ou d'imposer leurs propres solutions lors des séances de counseling. Quand les PVVIH tombent malades et meurent, l'ONG connaît une interruption de services et des coûts supplémentaires pour former les remplaçants, d'où un risque pour la viabilité à long terme de l'organisation elle-même. Dans certains cas, la visibilité fait de certaines PVVIH des sortes de "vedettes » avec toutes les tensions que cela entraîne au sein d'une organisation. Par ailleurs, des conflits peuvent survenir entre les PVVIH et le personnel séronégatif si le rôle et les contributions des uns et des autres ne sont pas estimés à leur juste valeur.

\section{Le contexte socioéconomique influence profondément la participation des PVVIH.}

Toute stratégie en vue de promouvoir une participation accrue des PVVIH dans les pays en développement doit tenir compte du contexte social, surtout les conditions de pauvreté, l'accès limité aux soins et aux traitements, l'inégalité entre les sexes ainsi que la stigmatisation et la discrimination. On remarque que la plupart des PVVIH qui participent dans les ONG impliquées dans l'étude proviennent de groupes à faibles revenus et doivent utiliser tout leur temps disponible pour gagner de l'argent, ce qui limite leur participation sur une base bénévole. Une mauvaise santé peut limiter la participation des PVVIH, bien que l'étude montre aussi que les PVVIH peuvent continuer à participer aux activités des ONG même quand elles sont symptomatiques, du moment qu'elles accomplissent des tâches qui tiennent compte de leur santé et pourvu qu'elles aient accès aux traitements. Les PVVIH, surtout les femmes et les personnes des groupes à faibles revenus, ont moins de chances d'avoir le niveau d'instruction nécessaire pour la prestation de services formels et la gestion de programmes. Dans certains endroits, l'analphabétisme est une barrière de taille entravant la participation. Les inégalités entre les deux sexes du point de vue de l'accès à l'éducation et aux services, des responsabilités ménagères, du fait qu'elles doivent s'occuper des enfants et qu'elles dépendent financièrement des hommes, empêchent bien des femmes séropositives de participer aux activités des ONG.

Si on ne donne pas l'argent pour le transport, seuls ceux qui sont vraiment motivés viennent. Quand ils savent qu'il existe un certain soutien, ils viennent. C'est probablement lié à la situation économique...il existe beaucoup de pauvreté, c'est difficile.

Membre d'ALAVI, Burkina Faso 
Nous ne sommes pas très instruits...parfois quand je dois parler j'hésite...je ne sais pas comment m'exprimer.

Bénéficiaire séropositive de CCDT, Inde

La crainte d'être stigmatisées et discriminées retient de nombreuses PVVIH de s'impliquer dans des ONG, notamment dans les organisations perçues comme des « associations de PVVIH » ou pour assumer des fonctions qui requièrent une visibilité importante. On le constate au Burkina Faso et en Inde ainsi qu'ailleurs en milieu rural où un nombre moindre de PVVIH sont visibles. Le rejet par la famille, les amis et la société ainsi que l'ignorance et la discrimination dans les services de santé et sur le lieu de travail alimentent les craintes de s'engager. En Equateur et en Zambie, certaines des personnes séropositives interviewées ont décrit des expériences de stigmatisation et de discrimination liées à leur participation visible aux ONG.

En Afrique...ce n'est pas facile. Si les gens disent que vous participez aux activités d'une organisation de personnes infectées, vous êtes marqué.

PVVIH, Burkina Faso

\section{Participer ne signifie pas forcément révéler sa séropositivité en public.}

Dans les quatre pays, l'étude a décrit une vaste gamme d'activités associatives menées par des PVVIH qui n'impliquent pas toutes la révélation de leur séropositivité en public. Par exemple, tous les PVVIH ne révèlent pas leur séropositivité à leurs clients pendant les séances de counseling ou au public présent lors des activités de prévention. Toutefois, on a également trouvé dans chaque pays des exemples de PVVIH qui témoignent en public sans se cacher et donnent ainsi un visage humain à l'épidémie, bien que très peu de PVVIH acceptent de parler de leur expérience dans les médias. En Zambie, c'est dans les activités de prévention que le plus grand nombre de PVVIH sont impliquées, contrairement aux trois autres pays, où seuls quelques individus font de la sensibilisation. La plupart des personnes interrogées ont noté qu'un certain niveau de participation visible de la part des PVVIH au sein d'une ONG sensibilise davantage aux points de vue et besoins des PVVIH et améliore les services. Toutefois, le niveau de visibilité dans les activités devrait dépendre du choix des PVVIH. Les PVVIH ne doivent pas se sentir forcées à révéler leur séropositivité et les ONG doivent garantir la confidentialité pour tous leurs prestataires de services et leurs bénéficiaires.

Après nous avoir vus, ils ont une autre perception de la vie. La peur les abandonne. Ils commencent à entrevoir qu'ils peuvent eux-aussi changer et vivre leur vie.

Conseiller séropositif, Armée du Salut, Inde

Les PVVIH craignent que les membres des associations ne découvrent leur séropositivité. Ils ont peur d'être marginalisés par la suite.

Prestataire de service, AMMIE, Burkina Faso

\section{La participation accrue des PVVIH requiert la mise en place d'un environnement un favorable dans les ONG.}

Les chercheurs ont constaté que le manque de ressources financières appropriées pour rémunérer les PVVIH était un grave problème pour les ONG dans les quatre pays. Cependant, un grand nombre d'ONG doivent également examiner leurs propres modes de fonctionnement, leurs politiques et les attitudes de leur personnel si elles souhaitent accroître la participation des PVVIH. Il existe des obstacles spécifiques entravant cette participation, notamment :

- Des attitudes paternalistes et des préjugés sur les PVVIH et leur participation de la part de nombreux professionnels de santé et travailleurs sociaux qui sont membres des associations.

Pour le moment, je ne pense pas que nous allons recruter une personne séropositive pour s'occuper des enfants.

Prestataire de services d'une ONG, Inde 
- Le manque de volonté institutionnelle et de politiques en vue de créer diverses possibilités et occasions de participer pour les PVVIH.

Les patients séropositifs ne participent au travail de notre organisation car nous n'avons pas de plans bien définis pour eux.

Prestataire de service séronégatif, Association Esperanza, Equateur

Ils m'ont dit que je pouvais venir et aider mais quand je suis arrivé, ils n'avaient aucune idée de ce que je pourrais faire.

Utilisateur de services séropositif, Equateur

- L'attention insuffisante portée aux questions de genre et d'orientation sexuelle. Le fait de penser, à tort, que toutes les PVVIH ont les mêmes besoins, quel que soit leur sexe ou orientation sexuelle, peut amener les ONG à offrir les mêmes services à tous leurs usagers, en passant à côté de certains besoins particuliers des femmes ou des HSH. Les personnes interviewées en Equateur et en Inde ont également mentionné les attitudes homophobiques de certains employés des ONG qui empêchent les HSH de participer aux activités de ces organisations ou de dire qu'ils sont homosexuels ou bisexuels quand ils participent.

Quand nous avons une réunion et que les mères viennent avec leurs enfants, je réalise combien il est important que les enfants aient un endroit où ils peuvent jouer pour que leur mère puisse se détendre.

Prestataire de services séropositive Dios, Vida y

Esperanza, Equateur

L'étude a identifié cinq catégories de facteurs institutionnels qui contribuent à la création d'un environnement favorable à la participation des PVVIH : attitudes du personnel, politiques et procédures non discriminatoires; formation ; soutien psychologique; soutien matériel et fonctionnement en réseaux. Ces facteurs sont développés dans la section des recommandations.

\section{Conclusions et recommandations}

Pour les PVVIH, il existe bien des manières de prendre part aux activités des ONG et les quatre types de participation identifiés-accès, inclusion, implication et participation accrue-englobent une vaste gamme de rôles, comme le montrent les résultats de cette étude réalisée dans quatre pays. Ce sont néanmoins l'accès et l'inclusion qui sont les types de participation observée dans les ONGles plus courants. La plupart des PVVIH participant à la prestation de services étaient des bénévoles, travaillant souvent de façon informelle et relativement peu d'entre eux étaient employés par les ONG dans des postes qualifiés. Toutefois, l'étude a mis en lumière un aspect important : tous les types de participation peuvent avoir un impact positif et une véritable participation des PVVIH n'implique pas

\section{Création et gestion de groupes d'auto-support}

Les groupes d'auto-support sont un volet important du soutien psychologique aux PVVIH car ils offrent un environnement non discriminatoire dans lequel les PVVIH peuvent partager leurs expériences et échanger des informations. Les ONG peuvent avoir recours aux mécanismes suivants pour renforcer les assises de tels groupes :

- Aider les membres à se fixer des attentes et objectifs communs.

- Chercher à faire participer les PVVIH dans une même région géographique, évitant ainsi des frais de transport qu'ils ne peuvent pas assumer.

- Clarifier les rôles et responsabilités des membres des groupes d'auto-support et des ONG, notamment au niveau de la prise de décisions.

- Mettre en place des mécanismes pour surmonter le décès des membres du groupe.

- Permettre aux membres des groupes de renforcer leurs compétences. 
forcément que les PVVIH révèlent leur séropositivité en public.

L'étude a également mis en exergue le fait que la participation des PVVIH aux activités des ONG relève d'un processus organisationnel. Si les ONG ne prennent pas certaines mesures indispensables, les effets positifs de la participation tendent à être de portée limitée et les conséquences négatives augmentent. Par exemple, si les PVVIH participent à des actions de prévention sans recevoir la formation et le soutien nécessaires, cela peut se répercuter sur la qualité des services et nuire aux PVVIH ellesmêmes.

Les ONG peuvent surmonter un grand nombre des obstacles qui entravent la participation en mettant en ouvre les recommandations suivantes :

\section{Promouvoir des attitudes et politiques positives et non discriminatoires à l'égard des PVVIH.}

- Evaluer les attitudes du management et des prestataires de services des ONG et leur apporter, le cas échéant, une formation les sensibilisant à ces questions.

- Examiner les politiques et modes de fonctionnement des ONG et supprimer ceux à l'origine de la discrimination à l'égard des PVVIH, notamment ceux qui affectent les femmes et les HSH.

- Garantir la confidentialité à toutes les PVVIH, tant le personnel que les usagers des services.

- Laisser aux PVVIH le choix de révéler ou non leur séropositivité.

\section{Renforcer la capacité de participation des PVVIH.}

- Donner des informations précises sur la prévention et le traitement du VIH/SIDA, ainsi que sur les services disponibles pour les PVVIH dans l'organisation et auprès d'autres prestataires de services.

- Utiliser les services de counseling comme point de départ pour développer les compétences des bénéficiaires.

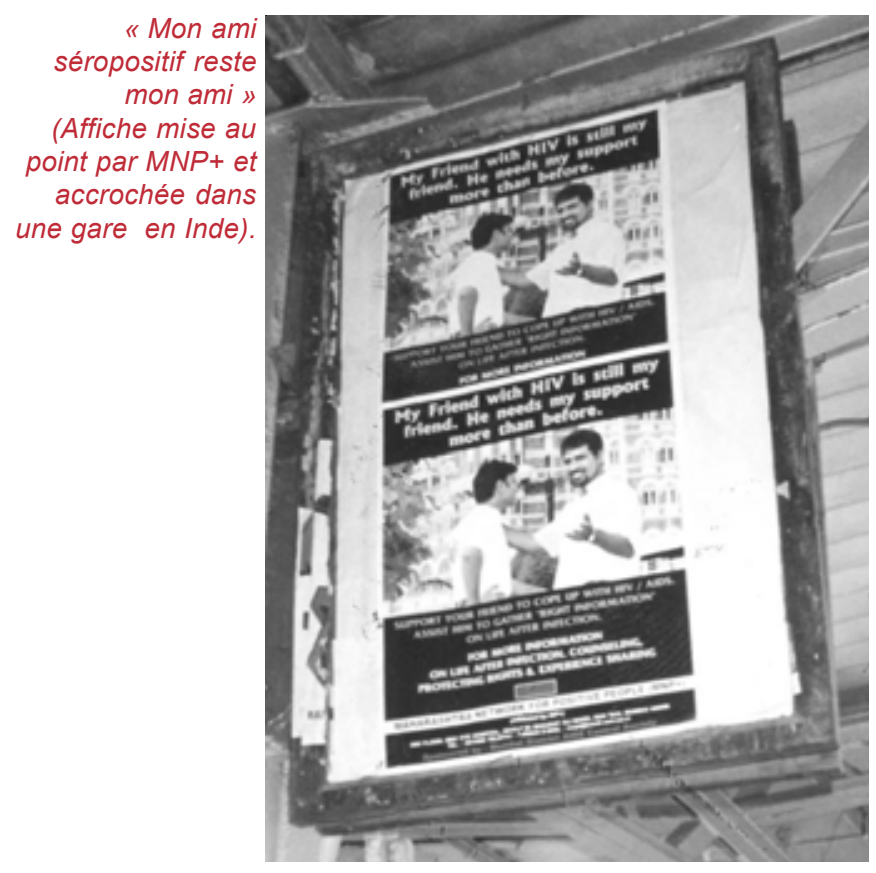

- Informer les PVVIH sur les possibilités de participation au sein de l'organisation et les orienter vers les activités correspondantes.

- S'assurer que les PVVIH reçoivent une formation gratuite ou abordable quand ils sont prestataires de services, comme tout autre employé ou volontaire.

- Prévoir un suivi individuel aux formations pour garantir des bénéfices à long terme.

\section{Apporter un soutien psychologique aux PVVIH et promouvoir l'auto-support.}

- S'assurer que la prise en charge assurée par les prestataires "professionnels » est de haute qualité et qu'elle est complétée par un soutien entre pairs.

- Offrir aux PVVIH bénéficiaires l'occasion de rencontrer d'autres PVVIH, soit par le biais du counseling par les pairs ou des groupes d'auto-support, en tenant compte du sexe et de l'orientation sexuelle.

- Assurer le counseling des PVVIH et de leur famille avant qu'ils ne donnent des témoignages en public.

- Mettre au point un counseling adapté pour aider les PVVIH à affronter les expériences de stigmatisation et de discrimination, qu'elles soient réelles ou vécues comme telles. 
Créer des réseaux avec d'autres organisations et institutions pour promouvoir la participation des PVVIH.

- Encourager les services de dépistage du VIH dans les secteurs public et privé pour qu'ils donnent des informations aux PVVIH sur les services offerts par les ONG et qu'ils les orientent vers les associations dans les meilleurs délais.

- Diversifier les activités des ONG et choisir soigneusement les stratégies de communication pour éviter que les organisations soient identifiées comme des « associations de PVVIH ».

- Travailler avec d'autres pour réduire la stigmatisation et la discrimination contre les PVVIH dans la société en faisant la promotion de la tolérance et de la compassion, en améliorant les connaissances sur le VIH/SIDA dans la société, en sensibilisant les leaders communautaires et religieux et en défendant les droits des PVVIH.

\section{Apporter un soutien matériel aux PVVIH qui ont peu de ressources.}

- Rémunérer les PVVIH en échange du temps passé à réaliser les activités et des compétences utilisées. Cela peut se faire sous forme d'argent, de nourriture, de médicaments, de soins médicaux, de remboursement des frais de déplacement ou de prise en charge des enfants suivant le type de participation.

- S'assurer que les PVVIH qui participent aux activités de l'association peuvent accéder à une prise en charge médicale gratuite ou à faible coût, notamment au traitement des infections opportunistes, en leur offrant une assurancemaladie, la possibilité d'utiliser les propres services de l'ONG ou en les orientant vers d'autres organisations ou services publics.
Equipes de recherche :

Alliance internationale contre le VIH/SIDA Royaume-Uni : Christophe Cornu (coordinateur international de l'étude et chercheur principal) ; Pam Decho (responsable du soutien du projet); Kathy Attawell (éditrice consultante)

Burkina Faso : Alfred Ouédraogo (coordinateur de la recherche) ; Gisèle Kaboré, Augustin Sankara, Soungalo Traoré (assistants de recherche)

Equateur : Doris Herrera et Norma Velasco (responsables de la recherche); Alejandrina Maldonado (assistante de recherche)

Inde : Rajiv Dua (coordinateur de la recherche); Vimla V. Nadkarni (chercheur principal/conseiller technique pour l'Inde); Bindiya Nimla (responsable de la recherche); Vaishali Sharma Mahendra (coordinateur Horizons/Population Council) au bureau régional Horizons/Population Council à Dehli ; Ujwala Bapat, Suchitra Inamdar, Neha Sachdev (assistants de recherche)

Zambie : Tashisho Chabala (responsable de la recherche); James Sulwe (coordinateur de la collecte de données) ; David Mwanza, Justina Namukombo, Eric Shimumbwe, Agatha Zulu Wachinga (assistants de recherche)

Etats-Unis-Horizons : Christopher Castle (Coordinateur Horizons/Alliance internationale pour le VIH/SIDA)

L'équipe du projet souhaite remercier les employés, les volontaires et les utilisateurs des services des 17 ONG qui ont participé à l'étude, INP+, NZP+, le personnel de l'IPC (Burkina Faso), Kimirina et CEPAR (Equateur), les bureaux du Population Council au Burkina Faso et en Zambie et les missions USAID.
Population Council/Horizons

4301 Connecticut Avenue, NW

Suite 280

Washington, DC 20008

horizons@pcdc.org

www.popcouncil.org/horizons
UsAID Cette publication a pu être réalisée grâce au soutien apporté par le Bureau Global de la Santé/VIH-SIDA, Agence ****** des Etats-Unis pour le Développement international aux termes de l'accord No. HRN-A-00-97-00012-00. Les opinions exprimées ici incombent aux auteurs et ne reflètent pas forcément les vues de l'Agence des Etats-Unis pour le Développement international.

Queensbury House 104-109 Queens Road Brighton, UK BN1 3XF mail@aidsalliance.org www.aidsalliance.org
๑ 2002 The Population Council Inc. 


\section{Vers une Participation Accrue des PVVIH AU BURKINA FASO}

$U$ ne année après l'achèvement de l'étude

diagnostique évaluant la participation des personnes vivant avec le VIH / SIDA (PVVIH) aux activités des O N G de lutte contre le SID A au Burkina Faso, une recherche a été effectuée pour déterminer l'impact sur les cinq O N G qui ont participé à l'étude et sur la politique nationale. D es chercheurs ont interviewé des membres des ON G, des utilisateurs des services et leurs familles pour juger si les $\mathrm{ONG}$ avaient réellement adopté les stratégies qu'elles avaient proposées et voir dans quelle mesure cela avait influencé la politique nationale.

\section{Résultats clés}

Les O N G ont lancé diverses activités pour réduire la stigmatisation des PVVIH au sein de leurs organisations.

Les O N G ont mis à jour les informations fournies lors des séances de conseils (counseling) pré et post-test. Elles ont sensibilisé leurs membres pour qu'ils changent leurs attitudes négatives envers les PVVIH et les ont encouragé à se soumettre au test de dépistage. Q uatre parmi les cinq ON G ont créé des groupes de parole pour les PVVIH et leurs familles et l'une d'entre elles dispose d'un groupe d'aide sociale qui apporte des repas aux $P V V I H$. Ces activités ont aidé les PVVIH à accepter leur statut, à se sentir solidaires et à partager leurs expériences sans s'exposer à des risques de discrimination. En revanche, les O N G expliquent qu'elles n'ont pas pu réduire les craintes des PVVIH d'être stigmatisées à l'extérieur des organisations. M algré l'organisation de formations pour encourager les PVVIH à participer à des réunions publiques d'information dans la communauté, celles-ci préfèrent faire du counseling individuel.

«I maginez que les personnes infectées et non infectées mangent ensemble dans le même plat, c'est une chose tellement positive. »PVVIH

\section{Plus de formations sont offertes aux PVVIH} mais le manque de compensation financière reste un obstacle à leur participation.

Toutes les O N G ont offert de nouvelles formations à leurs personnel et volontaires, notamment pour le counseling pré- et post-test, les visites à domicile, le suivi psychosocial, le plaidoyer et la prise en charge en général. L es volontaires séropositifs préfèrent de brèves séances de formation ou alors une formation prévue lors de leurs heures de travail car ils ont d'autres responsabilités qui ne leur laissent que peu de temps de libre. C'est l'O N G qui doit négocier leur disponibilité avec les employeurs sans pour autant compromettre le caractère confidentiel de leur séropositivité. L 'utilisation de volontaires est problématique tant pour les individus qui rencontrent bien des difficultés à travailler sans être payés que pour les organisations qui connaissent des interruptions de leurs services.

\section{Réseaux et partenariats ont permis aux O N G d'élargir l'accès à certains services.}

Les ON G souhaitant ouvrir de nouveaux centres de dépistage ont souvent été entravées par le manque de financement et l'insuffisance des trousses de test. Toutefois certaines des associations qui n'y avaient pas encore songé ont forgé des partenariats avec des prestataires de soins au niveau local pour élargir l'accès aux services de conseils et dépistage volontaires et à la prise en charge. D es accords ont été négociés avec des centres de dépistage, publics et privés, en vue de réduire les coûts du test en contrepartie du counseling offert par les associations. D eux O N G collaborent actuellement avec des médecins pour fournir des services de prise en charge et une $\mathrm{ONG}$ a mis en place un système de référence pour les PVVIH . En revanche, des partenariats avec des hôpitaux locaux n'ont pu être conclus pour élargir l'accès au traitement.

Les $\mathrm{ONG}$ ont commencé à faire connaître leurs activités au public : elles doivent continuer en ce sens.

Toutes les O N G ont cherché à faire connaître du public leurs activités et les possibilités de participation à leurs activités offertes aux PVVIH . En général, elles ont distribué des dépliants d'information et l'une des organisations a été présentée lors d'une émission radiophonique et télévisée. En revanche, d'autres stratégies ciblant des groupes intéressés, par exemple les journées portes ouvertes et les séances de discussion avec les PVVIH, n'ont pas été réalisées. 


\section{Impact au niveau national}

U ne fois achevée l'étude diagnostique, les O N G ont assisté à des réunions au niveau national portant sur la formulation des stratégies de lutte contre le VIH / SID A et elles ont pu partager avec le Programme national de lutte contre le SIDA et I'ONU SIDA les seules données disponibles sur les besoins des PVVIH au Burkina Faso. Les résultats de l'étude ont poussé à revoir les stratégies nationales de lutte contre le VIH/ SID A afin d'inclure les besoins des PVVIH au niveau de la prise en charge.

« Je suis un membre, je ne suis pas actif, je participe seulement aux réunions du groupe de parole. A part cela, on ne m'a pas dit que je pouvais participer à d'autres activités de l'association. » M embre d'un groupe de parole de PVVIH

\section{Peu d'avancées en ce qui concerne la participation des PVVIH à la gestion des O NG et à la prise de décisions.}

Certes, toutes les O N G ont cherché à accroître la participation des PVVIH à la prise de décisions.

Toutefois, seules quelques PVVIH savent qu'elles peuvent prendre part aux structures de gestion des organisations. $D$ 'après les personnes interrogées, les obstacles sont les suivants : structure hiérarchique des ON G et manque de précision quant aux possibilités de participer pour les PVVIH en dehors de l'utilisation des services.

\section{Participation accrue des femmes malgré la charge des responsabilités familiales.}

Les femmes sont à la tête de trois des O N G impliquées dans l'étude mais à part cela, la participation des femmes aux services des O N G reste limitée. Suite à l'étude diagnostique, certaines femmes ont pris en main des activités telles les kiosques d'information et une pharmacie. $D$ 'autres activités réservées exclusivement aux femmes ont été démarrées dont un groupe de parole et un projet d'activités génératrices de revenus. L'une des ONG oriente maintenant les femmes vers des services juridiques pour les problèmes de succession suite à un divorce lié au SI DA.

\section{Conclusions}

$C^{\prime}$ 'est le processus de recherche à caractère participatif de l'étude diagnostique qui a permis au personnel et aux membres des O N G d'envisager, souvent pour la première fois, les changements qu'ils peuvent faire dans l'organisation et les améliorations de leurs services grâce à la participation des PVVIH . L'étude de suivi a fait la constatation suivante : les $\mathrm{ON}$ G étaient plus à même d'adopter des stratégies de participation des PVVIH qui demandaient des changements organisationnels internes et peu de ressources, mais elles étaient trop ambitieuses en ce qui concerne les changements qui nécessitaient des apports externes.

Toutefois, l'étude montre que les O N G peuvent prendre plusieurs mesures importantes en vue d'une participation accrue des PVVIH, notamment des femmes. Les recommandations suivantes pour accroître la participation des PVVIH sont tirées de l'étude:

- Formuler des plans stratégiques réalistes avec des priorités d'amélioration stipulées clairement.

- Améliorer les compétences du personnel et des volontaires afin qu'ils puissent créer un contexte favorable à la participation des PVVIH .

- C réer des réseaux avec d'autres O NG et des structures de prise en charge afin d'élargir l'accès des PVVIH aux services.

J uillet 2003

Equipe de recherche : Lisanne Brown, Horizons/Tulane University ; Augustin Sankara, Gisèle Kaboré, Idrissa Ouédraogo, Lydia Tapsoba, consultants indépendants ; Joshua Volle, Tulane University. Hprizons

Population Council/Horizons

4301 Connecticut Avenue, NW Suite 280

Washington, DC 20008

horizons@pcdc.org

www. popcouncil.org/ horizons

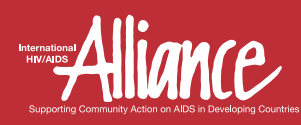

International HIV/AIDS Alliance

Queensbury House

104-109 Queens Road

Brighton, UK BN1 3XF

mail@ aidsalliance.org

www. aidsalliance. org
Horizons réalise des recherches opérationnelles à l'échelle mondiale dans le but d'améliorer les programmes de prévention et de prise en charge dans le domaine du VIH/SIDA. Horizons est mis en œuvre par le Population Council en partenariat avec I'International Center for Research on Women (ICRW), le Program for Appropriate Technology in Health (PATH), International HIV/AIDS Alliance, Tulane University, Family Health International et J ohns Hopkins University.

USAID Cette publication a pu être réalisée grâce au soutien apporté par le Bureau Global de la Santé/ VIH-SIDA, Agence des Etats-Unis pour le Développement $\star \star \star \star \star \star * *$ international aux termes de l'accord No. HRN-A-00-97-00012-00. Les

international aux termes de l'accord No. HRN-A-00-97-00012-00. Les
opinions exprimées ici incombent aux auteurs et ne reflètent pas forcément les vues de l'Agence des Etats-Unis pour le Développement international. 